\title{
Is There a Relationship between Serum D-Dimer Levels and Thorax CT Severity Score in Patients with COVID-19?: A Retrospective Analysis
}

\author{
Belma Cevik, MD* and Kayıhan Akın, MD \\ Department of Radiology, Lokman Hekim University School of Medicine, Turkey
}

*Corresponding author: Belma Cevik, Department of Radiology, Lokman Hekim University School of Medicine, Ankara, Turkey

\section{Introduction}

The COVID-19 pandemic began in Wuhan (Hubei, China) in December 2019 and rapidly spread around the World. Because of RT-PCR sensitivity limitations [1], the radiology literature has focused on detecting the probability of infection of non-contrast CT findings [2]. However the elevated serum D-dimer levels and its relationship with poor outcomes in COVID-19 pneumonia patients have been demonstrated [3]. D-dimer is known as a biomarker of inflammation. Therefore, we considered that serum D-dimer levels may predict the severity of inflammation. CT involvement extent is the most visual parameter, which could reflect the severity of inflammation. In this study, we investigated the relationship between D-dimer levels and thorax CT severity score in the patients with COVID-19.

\section{Methods}

In this retrospective study, 84 patients with COVID-19 pneumonia who were diagnosed with RTPCR and thorax CT findings and whose serum CRP and D-dimer levels were available were included. The study was performed in Lokman Hekim University between April 1, 2020 and December 31, 2020. The study was approved by the local ethics committee.

Serum D-dimer and CRP levels were obtained on admission. CRP and D-dimer levels were tested using immunoturbidimetric assay with the reference ranges of 0-5 $\mathrm{mg} / \mathrm{L}$ and 0-500 $\mathrm{ng} \mathrm{FEU} / \mathrm{mL}$, respectively. Thorax
CT examinations were performed with Toshiba Aquilion 64 multidetector CT scanner. Contrast material was not used. The tube voltage was determined as $120 \mathrm{kV}$ with automatic tube current modulation. The matrix size was $512 \times 512$. Thin reformat images with a slice thickness of $2 \mathrm{~mm}$ were reconstructed from the images taken with a slice thickness of $1 \mathrm{~mm}$ in the axial plane.

Thorax CTs were evaluated by two radiologists ( 12 and 18 years of experience) in terms of distribution of lesions and imaging findings. CT findings were evaluated for ground-glass density, consolidation, fibrous stripe, pleural effusion, mediastinal lymphadenopathy, ratio of lung involvement and, the severity score of thorax CT was calculated.

Radiologically, the severity of the COVID-19 pneumonia was classified into 4 groups. The area of affected lungs consistent with COVID-19 pneumonia in each patient's CT after admission was classified as; no affect (score 0 ), $\leq 30 \%$ (score 1 ), $31-50 \%$ (score 2 ), and $\geq$ $50 \%$ (score 3 ) of total lung area.

\section{Results}

We included 84 consecutive patients to our retrospective study. All patients were positive with RTPCR. Among patients, forty seven (55.95\%) were males and $37(44.05 \%)$ were females. Their ages ranged from 21-94 and the median age 54 years. Serum D-dimer level was normal in 47 patients. D-dimer elevation was detected in 37 patients. The median D-dimer level was

Citation: Cevik B, Akın K (2022) Is There a Relationship between Serum D-Dimer Levels and Thorax CT Severity Score in Patients with COVID-19?: A Retrospective Analysis. Int J Radiol Imaging Technol 8:089. doi.org/10.23937/2572-3235.1510089

Accepted: January 12, 2022; Published: January 15, 2022

Copyright: (C) 2022 Cevik B, et al. This is an open-access article distributed under the terms of the Creative Commons Attribution License, which permits unrestricted use, distribution, and reproduction in any medium, provided the original author and source are credited 
Table 1: The comparison of CT findings between the normal D-dimer group and elevated D-dimer group.

\begin{tabular}{|l|l|l|l|}
\hline Radiologic findings & Total & D-dimer $\leq$ & D-dimer \\
$\mathbf{n = 8 4}(\mathbf{\%})$ & $\mathbf{n = 4 7 ( \% )}$ & $\mathbf{n = 3 7 ( \% )}$ \\
\hline No affected lung lobe & $21(25)$ & $15(31.9)$ & $6(16.2)$ \\
\hline$\geq 2$ affected lung lobe & $52(61.9)$ & $25(53.1)$ & $27(72.9)$ \\
\hline Unilateral lung involvement & $11(13)$ & $6(12.7)$ & $5(13.5)$ \\
\hline Bilateral lung involvement & $51(60.7)$ & $29(61.7)$ & $22(59.4)$ \\
\hline Ground-glass opacity & $51(60.7)$ & $29(61.7)$ & $22(59.4)$ \\
\hline Consolidation & $18(21.4)$ & $6(12.7)$ & $12(32.4)$ \\
\hline Fibrous stripe & $29(34.5)$ & $12(25.5)$ & $17(45.9)$ \\
\hline Pleural effusion & $7(8.3)$ & $2(4.2)$ & $5(13.5)$ \\
\hline Mediastinal LAP ${ }^{1}$ & $14(16.6)$ & $6(12.7)$ & $8(21.6)$ \\
\hline
\end{tabular}

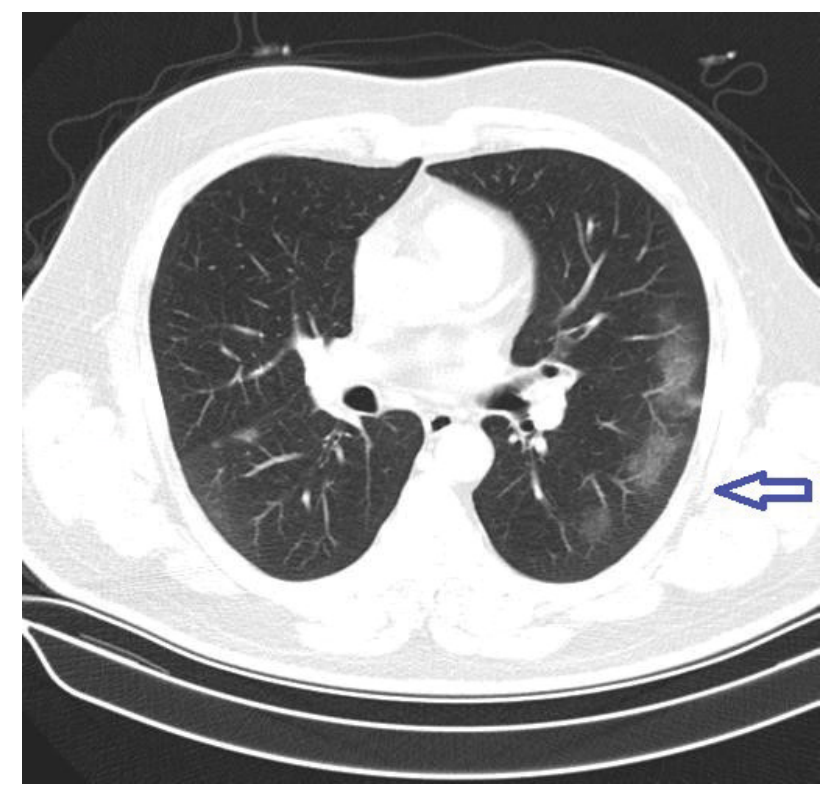

Figure 1: A 48-year-old male with COVID-19 pneumonia. Thorax CT images were in the parenchymal window (W: 1600-L: -600). CT images show ground-glass densities in the subpleural areas (arrow) of the left lungs (Score 1).

$1.145,79 \mathrm{ng} \mathrm{FEU} / \mathrm{mL}$ (43-10.186 $\mathrm{ng} \mathrm{FEU} / \mathrm{mL})$. Another inflammation marker, the median CRP level was 60.4 $\mathrm{mg} / \mathrm{L}$. Elevated CRP showed correlation with elevated D-dimer level in 32 patients.

Thorax CT results were obtained from 84 patients. $25 \%(21 / 84)$ of the patients did not have any lung involvement. Less than $30 \%$ lung involvement was determined in $45.2 \%(38 / 84)$ of the patients while 31 $50 \%$ lung involvement was present in $6 \%(14 / 84)$ of the patients and $>50 \%$ lung involvement was present in $13.1 \%(11 / 84)$ of the patients.

The predominant changes seen were ground- glass opacity in 51 patients (60.71\%), followed by patchy consolidation in 18 patients $(21.42 \%)$, fibrous stripe in 29 patients (34.52\%), pleural effusion in 7 patients (8.33\%) and mediastinal lymphadenopathy in 14 patients $(16.66 \%)$. Lung involvement was often bilateral (60.71\%). The comparison of CT findings between the normal D-dimer group and elevated D-dimer group are

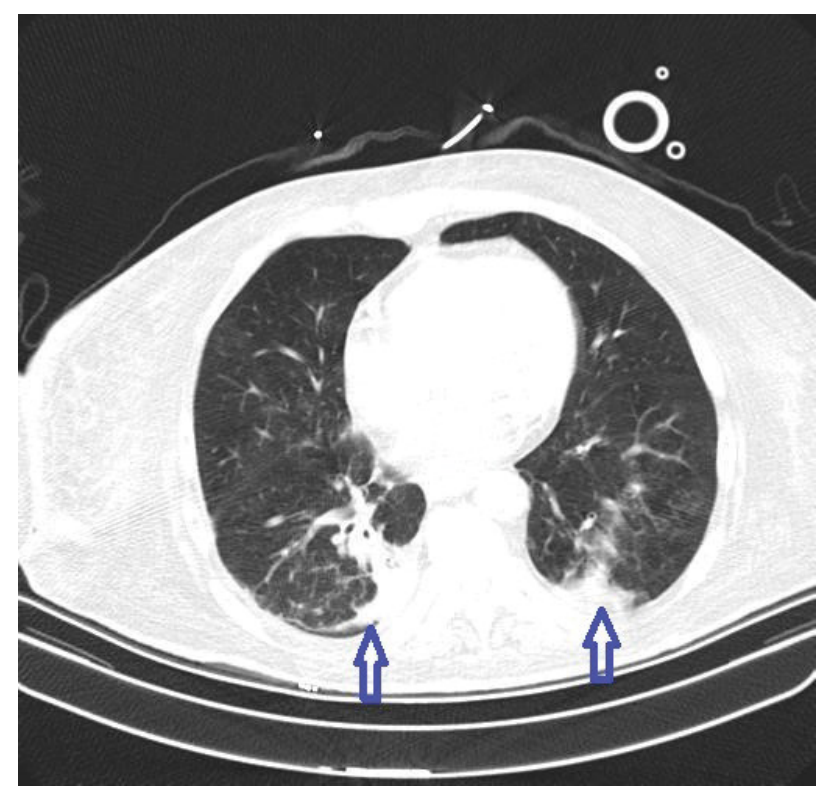

Figure 2: A 69-year-old female with COVID-19 pneumonia. Thorax CT images were in the parenchymal window (W: 1600-L: -600). CT images show consolidation and fibrous stripe in the both of lungs (Score 2).

\section{shown in Table 1.}

Thorax CT severity score was zero in 21 patients, one in 38 patients (Figure 1), two in 14 patients (Figure 2), and three in 11 patients (Figure 3). The comparison of $\mathrm{CT}$ severity score between the normal D-dimer group and elevated D-dimer group are shown in Table 2.

\section{Discussion}

We demonstrated that in patients diagnosed with COVID-19, serum D-dimer elevation upon admission was common and was associated with thorax CT severity score. D-dimer is a small protein fragment released in to the blood when a blood clot degraded by fibrinolysis. However, any pathologic or non-pathologic process that increases fibrin production or break down also increases plasma D-dimer levels. Examples include deep vein thrombosis, pulmonary embolism, arterial thrombosis, disseminated intravascular coagulation and conditions such as pregnancy, inflammation, cancer, chronic liver 

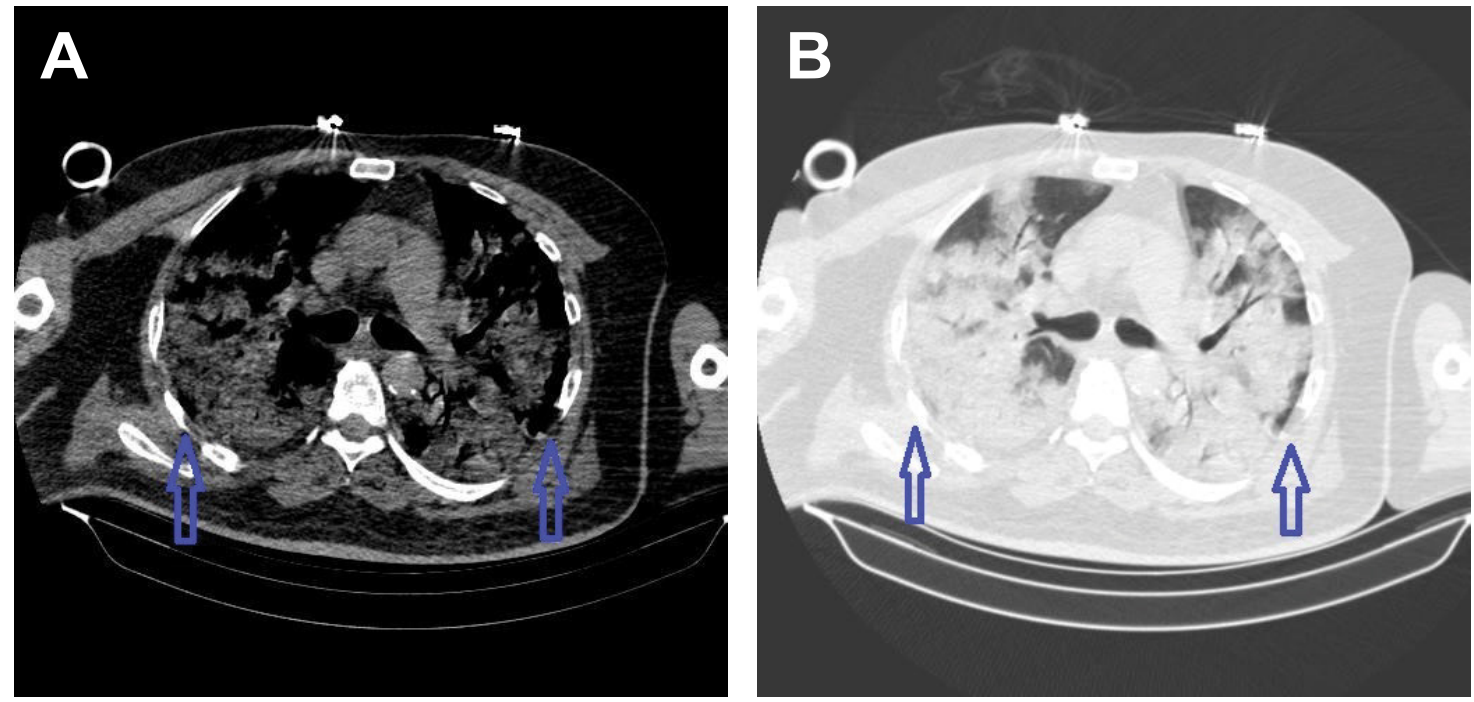

Figure 3: A 57-year-old female with COVID-19 pneumonia. Thorax CT images were in the parenchymal window (W: 1600 L: -600). CT images show diffuse consolidation (arrow) (Score 3) (A-B).

Table 2: The comparison of CT severity score between the normal D-dimer group and elevated D-dimer group.

\begin{tabular}{|l|l|l|l|}
\hline CT score & Total $(\mathbf{n}=\mathbf{8 4}) \mathbf{( \% )}$ & $\begin{array}{l}\text { Normal D-dimer } \\
\text { group } \mathbf{n}=\mathbf{4 7}(\mathbf{\%})\end{array}$ & $\begin{array}{l}\text { Elevated D-dimer } \\
\text { group } \mathbf{n}=\mathbf{3 7}(\%)\end{array}$ \\
\hline 0 & $21(25)$ & $15(31.9)$ & $6(16.2)$ \\
\hline 1 & $38(45.2)$ & $21(44.6)$ & $17(45.9)$ \\
\hline 2 & $14(16.6)$ & $6(12.7)$ & $8(21.6)$ \\
\hline 3 & $11(13.1)$ & $5(10.6)$ & $6(16.2)$ \\
\hline
\end{tabular}

diseases, post-traumatic, surgery status and vasculitis. D-dimer was not previously considered a biomarker in viral or bacterial pneumonia. Li, et al. and Zhou, et al. found that high mortality in patients with COVID-19 was due to the high level of D-dimer [3,4]. Tang, et al. reported that those with severe conditions had D-dimer values 3.5 times higher of the other patients [5]. Yilmaz, et al. reported that those with serum ferritine and D-dimer levels were observed to be high in the CTpositive group and have moderate positive correlation with CT severity [6].

A large body of clinical work investigating the abnormalities in laboratory parameters of COVID-19 disease documented high D-dimer, Ferritin, CRP values and low lymphocyte count [7]. We also measured at CRP levels and found elevated CRP showed correlation with elevated D-dimer level in our study. Özer, et al. reported that $\mathrm{CT}$ stage, CRP and D-Dimer values should be adopted as crucial prognostic factors in order to provide efficient healthcare [8].

COVID-19 is a newly identified viral disease that causes severe respiratory failure. Thorax $\mathrm{CT}$ is frequently used in the diagnosis of COVID-19 pneumonia, in the course of the disease, in demonstrating progression and residual disease. The stages of radiological recovery in patients with COVID-19 pneumonia were reported by Akçay, et al. [9]. In the initial phase (0-4 days), the main CT finding is ground glass density in the bilateral or unilateral sub pleural area. In the progressive stage (5-8 days), bilateral multi lobar distribution, diffuse ground glass densities, cobblestone pattern and consolidation are common CT findings. In the peak stage (9-13 days), consolidation, cobblestone pattern, diffuse ground glass density, and residual parenchyma fibrous bands are among the CT findings.

Our CT findings in COVID-19 patients were very similar to the another studies included predominantly peripheral ground-glass density, consolidation and fibrous stripes $[10,11]$. A previous study reported that lung involvement were bilaterally and predominantly right lung [12]. In our study, we found often lung involvement were bilaterally and predominantly right lung too. It should be kept in mind that D-dimer will be higher in patients with thorax $\mathrm{CT}$ involving more than 2 lobe, pleural effusion, mediastinally lymphadenopathy, consolidation and fibrous stripe. In these, care should be taken in terms of prognosis, treatment should be started early and followed closely.

This study has some several limitations. First, the current study was done in a single center. Second, it's a retrospective analysis and patients we included in the present analysis were not systematically assessed for the presence of pulmonary embolism. Further researches may be needed when extrapolated to wider patient population. 
In conclusion, we found that a high level of D-dimer in patients with COVID-19 was associated with thorax CT severity score. D-dimer may be a potential prognostic maker for the early warning severe cases. We believe that the findings are helpful for diagnosed the of COVID-19 pneumonia who showed elevated serum D-dimer. Especially, D-dimer levels measured at the time of admission to the hospital can be taken into consideration to predict radiological severity.

\section{References}

1. Xie X, Zhong Z, Zhao W, Zheng C, Wang F, et al. (2020) Chest CT for Typical Coronavirus Disease 2019 (COVID-19) Pneumonia: Relationship to Negative RT-PCR Testing. Radiology 296: E41-E45.

2. Prokop $M$, van Everdingen $W$, van Rees Vellinga $T$, van Ufford HQ, Stöger L, et al. (2020) CO-RADS: A categorical CT assessment scheme for patients suspected of having COVID-19-definition and evaluation. Radiology 296: E97-E104.

3. Akçay Ş, Özlü T, Yılmaz A (2020) Radiological approaches to COVID-19 pneumonia. Turk J Med Sci 50: 604-610.

4. Li J, Liu Z, Wu G, Yi M, Chen Y, et al. (2020) D-Dimer as a prognostic indicator in critically III patients hospitalized with COVID-19 in Leishenshan Hospital, Wuhan, China. Front Pharmacol 11: 600592.

5. Zhou F, YuT, Du R, Fan G, Liu Y, et al. (2020) Clinical course and risk factors for mortality of adult in patients with COVID-19 in Whuan, China: A retrospective cohort study. Lancet 395: 1054-1062.
6. Tang N, Li D, Wang X, Sun Z (2020) Abnormal coagulation parameters are associated with poor prognosis in patients with novel corona virus pneumonia. J Thromb Haemost 18: 844-847.

7. Yilmaz A, Sabirli R, Seyit M, Ozen M, Oskay A, et al. (2021) Association between laboratory parameters and CT severity in patients infected with Covid-19: A retrospective, observational study. Am J Emerg Med 42: 110-114.

8. Guan WJ, Ni ZY, Hu Y, Liang H, Ou CQ, et al. (2020) Clinical characteristics of corona virus disease 2019 in China. N Engl J Med 382: 1708-1720.

9. Özer KB, Çimenoğlu B, Özdemir A, Buz M, Koyuncu K, et al. (2020) The effect of radiological and laboratory parameters on prognosis in COVID-19 Disease. South Clin Ist Euras 31: $203-207$

10. Ai T, Yang Z, Hou H, Zhan C, Chen C, et al. (2020) Correlation of chest CT and RT-PCR testing in corona virus disease 2019 (COVID-19) in China: A report of 1014 cases. Radiology 296: E32-E40.

11. Caruso D, Zerunian M, Polici M, Pucciarelli F, Polidori T, et al. (2020) Chest CT features of COVID-19 in Rome, Italy. Radiology 296: E79-E85.

12. Espargallas I, Sevilla JJR, Chiaradia DAR, Salar A, Casamayor G, et al. (2020) CT imaging of pulmonary embolism in patients with COVID-19 pneumonia: A retrospective analysis. Eur Radiol 31: 1915-1922. 